\title{
Computational analysis of Alzheimer-causing mutations in amyloid precursor protein
} and presenilin 1

\section{Mehra, Rukmankesh; Kepp, Kasper Planeta}

Published in:

Archives of Biochemistry and Biophysics

Link to article, DOI:

10.1016/j.abb.2019.108168

Publication date:

2019

Document Version

Peer reviewed version

Link back to DTU Orbit

Citation (APA):

Mehra, R., \& Kepp, K. P. (2019). Computational analysis of Alzheimer-causing mutations in amyloid precursor protein and presenilin 1. Archives of Biochemistry and Biophysics, 678, [108168].

https://doi.org/10.1016/j.abb.2019.108168

\section{General rights}

Copyright and moral rights for the publications made accessible in the public portal are retained by the authors and/or other copyright owners and it is a condition of accessing publications that users recognise and abide by the legal requirements associated with these rights.

- Users may download and print one copy of any publication from the public portal for the purpose of private study or research.

- You may not further distribute the material or use it for any profit-making activity or commercial gain

- You may freely distribute the URL identifying the publication in the public portal 


\title{
Computational analysis of Alzheimer-causing mutations in amyloid precursor protein and presenilin 1
}

Rukmankesh Mehra and Kasper P. Kepp*

DTU Chemistry, Technical University of Denmark, DK-2800 Kongens Lyngby, Denmark

*Corresponding author. E-mail: kpj@kemi.dtu.dk. Phone: +045 45252409

\begin{abstract}
Single-point mutations in the genes coding for amyloid precursor protein (APP) and presenilin 1 (PS1), the active subunit of $\gamma$-secretase that cleaves APP to produce A $\beta$, are the main causes of rare but severe familial Alzheimer's disease (fAD). Recent structures of the transmembrane parts of APP and $\gamma$-secretase with a fragment of APP bound enable us to study the origins of the pathogenicity of the single amino acid changes in the context of the actual enzyme-substrate complex, which has not previously been possible. We used the new structures as input for several state-of-the-art computational methods that predict the folding stability effect of mutations. We find that pathogenic mutations almost exclusively reduce the stability of the proteins. Since most "random" mutations of an evolutionarily optimized protein tend to destabilize, we also show that the APP mutations destabilize the complex-bound substrate more than the free substrate, indicating reduced affinity of APP to $\gamma$-secretase. We confirmed this using two other methods, BEATMUSIC and mCSM PPI, specifically developed for calculating binding affinities of mutants. Although pathogenic PS1 mutations destabilize the complex and substrate-free form to the same extent, they significantly destabilize the protein more than the control set of random mutations. We conclude that fAD mutations most likely reduce the stability of the protein-substrate complex and thus retention time of APP-C99, leading to premature release of longer toxic $A \beta_{42}$ in accordance with the FIST model of $A \beta$ production, whereas the observed general destabilization of the protein may reduce activity towards other substrates.
\end{abstract}

Keywords: Alzheimer's disease; presenilin; $\gamma$-secretase; mutations; protein stability 


\section{Introduction}

Alzheimer's disease (AD), the major irreversible neurodegenerative disease that is the main cause of dementia, remains without a cure due to its molecular complexity[1-3]. More than 50 million people are estimated to suffer from dementia, with more than half of these cases being due to $\mathrm{AD}[4,5]$. Particular growth in prevalence is projected for developing countries with rapidly expanding elderly populations such as India and China[6].

$\gamma$-secretase, a membrane protease complex, cleaves the transmembrane domain of the Cterminal fragment of the amyloid precursor protein (APP-C99) to produce $\beta$-amyloid (A $\beta$ ) peptides of varying length, which are currently considered culprits of the disease according to the amyloid hypothesis[7-11]. $\gamma$-secretase consists of four subunits: Nicastrin, presenilin (either PS1 or PS2), APH-1, and PEN-2[12,13]. Presenilin is the catalytic subunit[14,15] and APP is one of its many substrates (Figure 1), which also include Notch[16]. More than 200 mutations have been identified in PS1 and nearly 50 mutations in APP that are potentially related to $\mathrm{AD}[17,18]$. Of these, 149 and 17, respectively, are pathogenic missense mutations in the transmembrane (TM) regions of PS1 and APP. These mutations affect the $\gamma$-secretase activity[19], change the amount of $A \beta$ formed and specifically increase the $A \beta_{42} / A \beta_{40}$ ratio[2022]. Since $A \beta_{42}$ is more aggregation-prone[23] and since the $A \beta_{42} / A \beta_{40}$ ratio correlates with disease onset (using the data by Sun et al. but removing the extreme loss-of-function outlier at the catalytic site)[24], this molecular effect remains a current hypothesis for the function of AD-causing mutations.

$\gamma$-secretase has a remarkably slow turnover of substrates, with an estimated $\mathrm{k}_{\text {cat }}$ of 0.0012 $\mathrm{s}^{-1}$ and a surprisingly large $\mathrm{K}_{\mathrm{M}}$ of $0.2 \mathrm{mM}$, giving an extremely slow turnover of $6 \mathrm{~s}^{-1} \mathrm{M}^{-1}$.[25] The large $K_{M}$ is probably related to an unusually small and conformation-dependent substrate affinity, which can be further impaired by mutations if these destabilize the protein[26]. According to the FIST (Fit-(Induce)-Stay-Trim) mechanism[27,28], the slow and sloppy intramembrane protease works by a gradual fitting and squeezing of the substrate within the active site "fist", such that stronger "grapping" (stability of the complex) leads to a longer retention and additional squeezing into shorter products, whereas loose grapping (impaired substrate affinity) leads to imprecise cleavage and premature release of products. The consistent increase in the $A \beta_{42} / A \beta_{40}$ ratio[29] indicates that the change in substrate cleavage is related to pathogenicity. According to the FIST model, $\gamma$-secretase modulators work to stabilize the complex and thus lower the $A \beta_{42} / A \beta_{40}$ ratio[30] whereas fAD mutations destabilize the complex and thus increase the ratio[27,28]. 
We hypothesize here that the pathogenic APP and PS1 mutations work in one of two ways: either by decreasing the binding affinity of APP-C99 to $\gamma$-secretase (the enzyme-substrate complex) or by generally decreasing the stability of the compact membrane protein, as has been hypothesized from sequence-based computations[28]; such stability loss may lead both to loss of function of the protein[31,32], as well as gain of toxicity by elevating the cost of protein turnover of $\gamma$-secretase[33]. To explore these two options we need to investigate as many mutations as possible, we need the structures of the complex and enzyme and substrate alone, and we need control data sets of mutations that are not pathogenic. Recently, two $\gamma$-secretase cryo-electron microscopy structures were elucidated with C83 (a shorter analog of APPC99)[34] and Notch as substrates[35]. These structures for the first time enable a comparison of stability changes upon mutation between the substrate-free and the substrate-bound states of $\gamma$-secretase and thereby indirectly, the impact of mutations on the substrate binding affinity. The present paper represents an attempt to perform this analysis.

We studied the change in stability ( $\Delta \Delta \mathrm{G}_{\mathrm{MUT}}$ ) caused by APP and PS1 mutations in the transmembrane (TM) region, where structural coordinates are available, in both the apo- and holo- $\gamma$-secretase structures (Figure 1d and 1e), To enable a statistical test of the significance of the computations, we generated data sets with 202 and 3048 "random” mutations in variable sites of the APP-C99 fragment and PS1, respectively, for both apo- and holo-structures. We used the recently published structure 6IYC[34], because it contains all the subunits including the TM part of the substrate analog C83, and the TM part of PS1. $\Delta \Delta \mathrm{G}_{\mathrm{MUT}}$ was studied using four well-known methods; FoldX[36,37], POPMUSIC[38,39], mCSM[40], and I-Mutant[41].

All the methods, despite their diversity and variations in structural input, show that pathogenic mutations in both APP-C99 and PS1 markedly and consistently reduce the stability of the proteins. Since mutations tend to be destabilizing in general, we also compared the mutation effects to test sets of "random" mutations. We find that pathogenic APP-C99 mutations consistently decrease stability more in the holo-structure than in the apo-structure (PDB ID: 2LLM) indicating that they decrease the binding affinity of the APP-C99 substrate. We confirmed this finding by using two methods (mCSM-PPI and BEATMUSIC) that directly calculate the change in binding affinity $\left(\Delta \Delta \mathrm{G}_{\text {bind }}\right)$ of the enzyme-substrate complex upon mutation. The pathogenic PS1-TM mutations consistently destabilize both the apo- and holostates significantly more than a control dataset of random mutations. Our computations support a mechanism whereby fAD mutations reduce the stability of the active proteins, but do not determine whether the effect on $A \beta$ or another substrate (e.g. Notch) is the cause of disease. 
(a) APP transmembrane apo (2LLM)

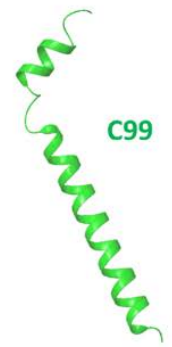

(d) APP transmembrane domain 2LLM 686 QKLVFFAEDVGSNKGAIIGLMV 6IYC_E 686 - - LVFFAE - - - - KGAIIGLMV 2LLM 708 GGVVIATVIVITLVMLKKK 726 6IYC_E 708 GGVVIATVIVITLVMLKKK 726

(e) PS1 sequence (PDB: 6IYC_B) (b) $\mathrm{v}$-secretase apo (5FN2)

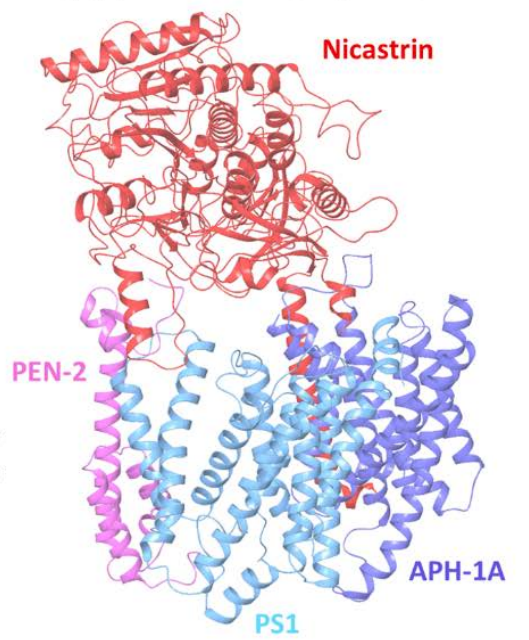

(c) Y-secretase holo (6IYC)

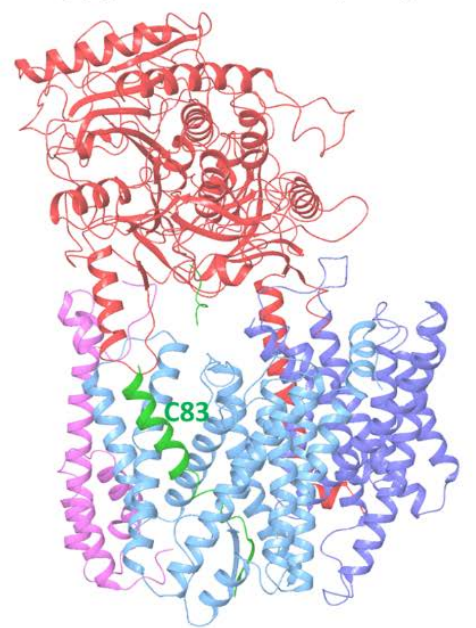

73 LTLKYGAKHVIMLFVPVTLCMVVVVATIKSVSFYTRKDGQLIYTPFTEDTETV GQRALHSILNAAIMISVIVVMTILLVVLYKYRCYKVIHAWLI 168 ISSLLLLFFFSFIYLGEVFKTYNVAVDYITVALLIWNFGVVGMISIHWKGPLRLQQAYLIMISALMALVFIKYLPEWTAWLILAVISVYDLVAVLCP 265 KGPLRMLVETAQERNETLFPALIYSST 291376 ERGVKLGLGDFIFYSVLVGKASATASGDWNTTIACFVAILIGLCLTLLLLAIFKKALPALPI 438 SITFGLVFYFATDYLVQPFMDQLAFHQFYI

Figure 1. Experimental structures used in this study. (a) 43-residue NMR model of the transmembrane region of APP-C99 (PDB code: 2LLM). (b) Substrate-free apo- $\gamma$-secretase structure (5FN2). (c) APP-C83-bound holo- $\gamma$-secretase structure (6IYC). (d) APP sequence of the structural elements present in 2LLM and 6IYC (chain E) with TM mutations highlighted in red. (e) PS1 sequence of the residues present in 6IYC (chain B) with TM mutations highlighted in red. 


\section{Methods}

\section{Structures used}

We used three PDB structures: 2LLM[42], 6IYC[34] and 5FN2[43]. 2LLM is a NMR structure containing 43 residues of the transmembrane part of free APP-C99 (named here APP-C99apo). 6IYC is the recently published electron microscopy structure of $\gamma$-secretase complexed with a 83-residue (C83) analog of APP-C99 (named holo); it contains 34 residues of APP-C83 as two fragments. 5FN2 is $\gamma$-secretase without any bound substrate (named PS1-apo). In the holo-structure 6IYC, APP-C99-holo refers to the TM part of C83 bound to $\gamma$-secretase, whereas PS1-holo refers to the structure of the PS1 subunit in the holo-structure.

In order to describe both the structures of the substrate APP-C99 before and after binding to $\gamma$-secretase (i.e. the apo- and holo-states), we performed all APP-C99 mutations both on PDB structures 2LLM[42] (APP-C99-apo) and 6IYC[34] (APP-C99-holo; Figure 1a and 1c). We used the experimental structures directly without any addition of missing residues, to avoid disturbing the experimental geometries. We used the top-listed NMR model of 2LLM for computations. 6IYC contains two mutations, Q112C in PS1 and V695C in APP-C83, although the fragment containing V695C is not visible in the structure. To model the WT protein, we converted the Q112C mutant of 6IYC into the WT (i.e. C was changed to Q at position 112) and geometry-relaxed the sidechain using Maestro. Both structures contain all the ten studied transmembrane mutated sites of APP-C99 associated with AD (Figure 1d, highlighted in red).

The most complete experimental PS1 WT apo-structure, 5FN2[43], was used for computing the stability effects of the PS1 mutations. Bai et al. have described several different apo-structures: 5FN3 has an ordered TM2 segment of PS1, which is partly absent in other structures[43]. A poly-alanine chain of 24 residues resides at the substrate-binding site. 5FN4 and 5FN5 have a disordered, invisible TM2 of PS1, and no ligand bound. The orientation of TM6 is different in all these three structures. The structure with a dipeptidic inhibitor $N$ - $[N$ (3,5-difluorophenacetyl)-L-alanyl]-S-phenylglycine $t$-butyl ester (DAPT; PDB ID: 5FN2) is very similar to 5FN3, with a C $\alpha$ RMSD of $0.4 \AA$ for the PS1 subunit; it is the most complete apo structure and includes more of TM6 than other apo-structures, the linker between TM2 and TM1/TM3, the cytoplasmic ends of TM3 and TM6, and part of the large hydrophilic maturation loop between TM6 and TM7[43]. The other published apo-structure of $\gamma$-secretase 5A63[13] misses TM2 and part of TM6, both of which interact strongly with the substrate, and thus these structures are not very useful for estimating mutation effects on substrate binding. 
PS1 in 5FN2 and 5A63 contains 301 and 215 residues, respectively, whereas PS1 in the holo-structure 6IYC contains 311 residues. 5FN2 has positions that represent 147 of the known PS1-TM fAD mutations, whereas 5A63 only contains positions covering $111 \mathrm{fAD}$ mutations. Due to the better structural coverage of mutation sites in 5FN2, we used this structure as the primary PS1-apo-structure for our calculations, whereas the substrate-bound state of PS1 in 6IYC was used as the holo-structure. We observed a Y256T mutation in PS1 of 5FN2, which we converted to the WT residue (Y) before calculation.

We used two approaches for the APP-C99 calculations: Using directly the experimental PDB structures (after conversion to wild type as described above) or using curated PDB structures. In the curated structures, missing side chains and hydrogen atoms were added, proper protonation states and bond orders were assigned, terminal residues were corrected and heteroatoms (in 6IYC, cholesterol, $\beta$-D-mannose, $N$-acetyl- $D$-glucosamine and 1,2-diacyl-Snglycero-3-phosphocholine) were removed using the Protein Preparation Wizard program of the Schrodinger software suite[44]. Most of the protein stability programs can accept both direct and curated PDB structures; therefore, to study the effect of protein preparation on stability changes, we used both the direct and curated structures.

\section{Membrane-protein system}

In order to identify pathogenic transmembrane mutations in APP-C99 and PS1 interacting with membrane, we built a protein-membrane system using the 6IYC holo-structure. A homogenous POPC lipid bilayer was built around 6IYC with a water thickness of $20 \AA$ using Position Protein in Membrane server[45] and CHARMM-GUI[46]. The C83 and PS1 residues forming direct contact with POPC were identified.

\section{Data sets of pathogenic mutations}

We used two datasets comprising the reported pathogenic missense mutations in the transmembrane (TM) region of APP and PS1 causing fAD (Supplementary Tables S1 and S2)[17,18]. The APP dataset (Table S1) consists of 17 TM mutations with association to AD and 1 TM mutation (L705V) associated with Cerebral Amyloid Angiopathy (CAA), which relates closely to $A D[47,48]$. The $A \beta_{42} / A \beta_{40}$ ratios for the APP dataset were compiled from Alzforum[17] and Alzheimer Disease \& Frontotemporal Dementia Mutation Database[18]. The PS1 dataset included 149 mutations in the TM region of the PS1 subunit of $\gamma$-secretase 
(Supplementary Table S2). This PS1 transmembrane mutation dataset was compiled from Tang et al.[49] and the $A \beta_{42} / A \beta_{40}$ ratios were compiled from Sun et al.[29]

\section{Control data sets}

Generally, we advocate a method of testing disease hypotheses by computing the change in chemical properties due to mutation both in a data set of pathogenic mutations and in one or more control sets of non-pathogenic or "random” mutations of the same protein in a student's t-test or ANOVA[50]. The best such control set consists of a significant number of clinically confirmed non-pathogenic mutations. In the common case where these are too few in number to constitute a statistically valid control set, one needs to define instead a data set of "random" mutations. It is however not straightforward and something that has concerned us for a while. As studies of the biophysical effect of pathogenic mutations become more and more common, this issue becomes increasingly important.

If confirmed non-pathogenic mutations are unavailable at necessary coverage (i.e. $<10$ mutations), we consider three other test sets: The largest set includes the computed property change for all possible mutations in the protein (saturated computational mutagenesis[50-52]). It is the most non-arbitrary test set and gives the best counting statistics but suffers from a bias towards mutations that are unlikely to occur and may reflect other mechanisms than the one being tested, including mutations in sites that never vary in the wild. A second choice of test set is that of saturated mutagenesis of all sites in which pathogenic mutations have been reported, excluding the pathogenic mutations themselves. This test set ensures that the sites used for control are actually variable and thus likely to produce functional proteins.

A third test set, which we use here, considers all mutations in sites that are evolutionarily variable by sequence alignment to homologs, to avoid the bias towards pathogenic behavior that is potentially associated with the pathogenic sites. To perform such a test, we searched the Conserved Domain Database [53] (CDD; Supplementary Figures S1 and S2) for PS1 and APP domain models. CDD contains well-annotated multiple sequence alignment models for domains and full proteins using sequences of related orthologs. The search was done using CDD version 3.17 (52910 PSSMs) database (E-value threshold 0.01), which contains NCBIcurated domains and data from the Pfam, COG, SMART, TIGRFAM and PRK databases. We analyzed the alignment of the CDD domain models for APP (UniProt ID: P05067) and PS1 sequences (UniProt ID: P49768) and studied the specific hit models as shown in Figures S1 
and S2. The control set was constructed by excluding the highly conserved sites (in red), and keeping the remaining sites as the evolutionary variable sites (blue); for these sites, all possible mutations were computed (19 for each site) and used as data points in the control set.

We performed computational saturation mutagenesis of all the 34 residues common to both 2LLM (APP-C99-apo) and 6IYC (APP-C99-holo), producing 646 mutations (18 pathogenic and 628 other mutations) in each state for a total of 1292 mutations. Similarly, saturation mutagenesis of all the residues common to both 5FN2 (PS1-apo) and 6IYC (PS1-holo) generated 5719 different mutations for each structure. Of these, 147 mutations were pathogenic and 5572 were all other possible mutations in the sites common to the two structures.

To enable use of the third and most accurate test, the highly conserved residues in both APP and PS1 were identified using CDD [53] (Supplementary Figures S1 and S2). These residues along with the pathogenic mutations were removed from the total set of possible mutations to form the control data set of random variable site mutations. These control sets included 202 and 3048 mutations for APP-C99 and PS1, respectively.

\section{$\Delta \Delta G_{M U T}$ calculations}

To ensure that our results are not sensitive to method choice, we used four well-known and diverse methods to calculate the folding free energy change due to mutation, $\Delta \Delta \mathrm{G}_{\mathrm{MUT}}$ in both apo- and holo-states of APP-C99 and PS1: FoldX[36,37], POPMUSIC 2.1[38,39], mCSM Stability[40], and I-Mutant3.0.[41,54] We also used two additional methods, mCSM-PPI[40] and BEATMUSIC[55], that directly calculate the change in binding affinity of APP-C99 to $\gamma$ secretase upon mutation (in 6IYC) and were specifically developed for these purposes. IMutant3.0 calculations were performed using binary classification; all methods used default setup. All the $\Delta \Delta \mathrm{G}_{\mathrm{MUT}}$ values are reported here in $\mathrm{kcal} / \mathrm{mol}$ and their sign conventions were made uniform for comparison such that $\Delta \Delta \mathrm{G}_{\mathrm{MUT}}<0$ represents an increase in the folding stability and $\Delta \Delta \mathrm{G}_{\mathrm{MUT}}>0$ represents a decrease in the stability.

To test the sensitivity of the calculated $\Delta \Delta \mathrm{G}_{\mathrm{MUT}}$ values of the APP-C99 mutations to reasonable changes in the protein structure, we used both the direct experimental PDB structures without any modifications (except adding the WT residue in 6IYC), and the curated PDB structures as discussed above. In addition, the FoldX calculations were performed both with and without the repair function to analyze the impact on results of curating the structure files used for input. The reported $\Delta \Delta \mathrm{G}_{\mathrm{MUT}}$ value from FoldX was the average of five runs. 
Saturation mutagenesis was performed only on the curated structures, as we did not observe any major difference between curated and direct structures (as discussed in results below). In order to compare the methods for the same structures consequentially, we used the same curated structures from Protein preparation wizard as input for all the methods.

In order to compute $\Delta \Delta \mathrm{G}_{\mathrm{MUT}}$ of the PS1 mutations, we also used the curated structures for all the calculations, and FoldX was used without any further repair function with one run, as we did not observe any major differences, neither when using the repair function on the curated structures nor when changing the number of runs in APP-C99. The average standard deviation of five runs (using FoldX without repair) for the 18 pathogenic mutations in free APP-C99 (2LLM) and holo APP-C99 (6IYC) were 0.1 and $0.4 \mathrm{kcal} / \mathrm{mol}$. Similarly, for the control set of 202 mutations, the average standard deviation of five runs was low, i.e. 0.12 and $0.29 \mathrm{kcal} / \mathrm{mol}$ for free APP-C99 (2LLM) and holo-APP-C99 (6IYC), substantially less than the computational uncertainty in these methods. 


\section{Results and discussion}

\section{Pathogenic mutations decrease the thermodynamic stability of APP-C99}

The experimental PDB structures provide the topology of the membrane proteins but miss many residues, display in some cases incorrect bond orders and mutations, and the residues lack hydrogen atoms and thus realistic protonation states. As these features will affect enzymesubstrate hydrogen bonding, a test of the sensitivity of structure-function relationships to reasonable variations in structural input is necessary. Accordingly, we analyzed the impact of structural curation by performing $\Delta \Delta \mathrm{G}_{\mathrm{MUT}}$ calculations on APP-C99 using both the direct and the curated PDB structures as described in Methods. As seen in Figure 2, we obtained similar results with both types of structural input, with positive values implying destabilizing effects. Although hydrogen bonding changes considerably upon curation, the $\Delta \Delta \mathrm{G}_{\text {MUT }}$ depends mainly on the chemical properties of the amino acids and the local polarity in the mutated site.

Using the experimental PDB structures directly, three of the four methods (POPMUSIC, mCSM Stability and I-Mutant; Figure 2a), show a clear consensus towards less stability of APP-C99 mutants. POPMUSIC produced a very small stabilizing effect for two mutations, T714A and T714I, which is however insignificant compared to the uncertainty in the methods of perhaps 1 kcal/mol[56-60]. FoldX displayed mixed results for the free apo-APP-C99 model (2LLM) but showed a clear overweight of destabilization in the holo-structure (6IYC), with exceptions being within the computational uncertainty. Comparing the left (apo) and right (holo) sides of Figure 2a, we see a consensus among these diverse methods that pathogenic APP-C99 mutations in particular destabilize the enzyme-substrate complex.

When using the curated structures with side chains of the missing residues and hydrogens added, we observe largely similar results (Figure 2b). Importantly, the FoldX results become much more in line with the results of the three other methods after curation, in particular for the apo-APP-C99 state (2LLM). Using FoldX with or without the recommended repair function of this program also produced similar $\Delta \Delta \mathrm{G}_{\mathrm{MUT}}$ energies (Figure 2a, 2b). Thus, reasonable variations in the structural input by accounting for hydrogen bonds and missing residues do not change our conclusion that the folding stability is impaired by the APP-C99 mutations.

To further test that our conclusions are robust towards reasonable variations in structural input, the $\Delta \Delta \mathrm{G}_{\mathrm{MUT}}$ energies computed on the experiment and curated structures show high correlation for both apo-APP-C99 (2LLM) and holo-APP-C99 (6IYC) states (Figure 2c). We conclude that for the overall data set, reasonable variations in structure (including the effect of mutation and hydrogen bonds in the experimental structures) do not affect conclusions, 
although interpretation of individual sites close to the mutated sites may be affected. For consistency, and because the experimental structures refer to mutants, we report results for our curated PDB structures in the remaining part of the work.

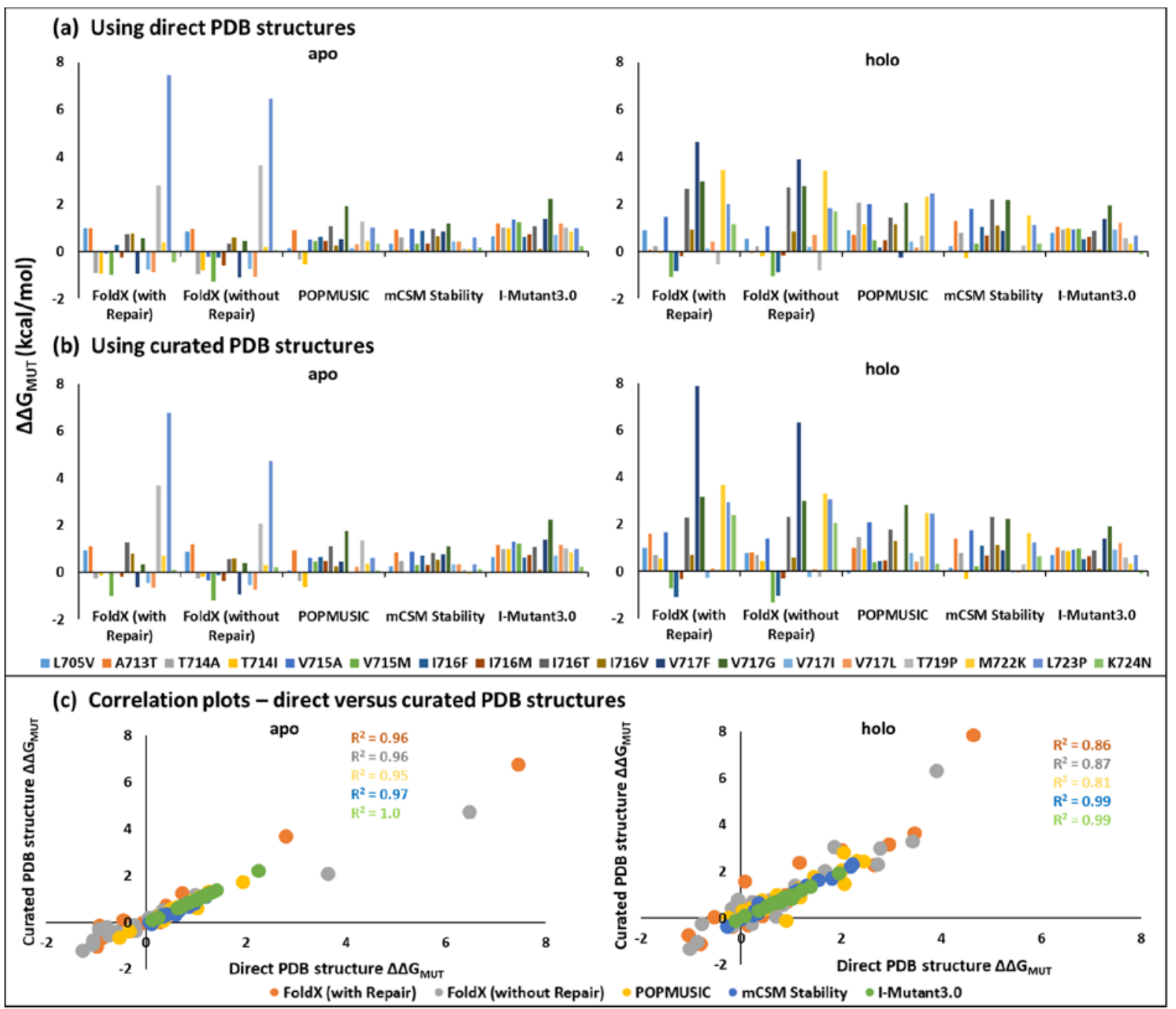

Figure 2. $\Delta \Delta$ GMUT $_{\text {energies calculated for apo-APP-C99 (2LLM) and APP-C99 bound to }}$ $\boldsymbol{\gamma}$-secretase (holo-APP-C99, 6IYC). a) Results using either the experimental structures directly. b) Results using the curated PDB structures as described in the Methods section (positive values represent destabilization). c) Correlation between $\Delta \Delta \mathrm{G}_{\mathrm{MUT}}$ energies computed using experimental and curated PDB structures for free APP-C99 (2LLM) and bound APPC99 (APP-C83; 6IYC).

To investigate the structural sensitivity of the methods and the differences between the apo- and holo-structures, we plotted the $\Delta \Delta \mathrm{G}_{\mathrm{MUT}}$ energies of the mutations in apo- versus holo- 
structures (Supplementary Figures S3-S7). The pathogenic datasets for APP-C99 and PS1 include 18 and 147 mutations and the control data set of all possible variations for the affected sites of APP-C99 and PS1 included 628 and 5572 mutations. FoldX was found to be highly sensitive to the structure input with small $\mathrm{R}^{2}$-values of correlation between apo- and holo-free energy changes.

In contrast, I-Mutant3.0 showed very high $\mathrm{R}^{2}$, indicating its small structure sensitivity as documented before[61]. POPMUSIC and MCSM displayed intermediate sensitivity. The wide differences in structural sensitivity confirms that the four methods are very distinct and that their combined use, if they agree, will make results much more significant than results based on only one or two methods, which generally cannot be trusted for the reasons shown in this analysis. This sensitivity also reflects that the apo-APP-C99 (2LLM) and holo-structures (6IYC) are very different, and much more different for the average amino acid than is the case for the apo- and holo-PS1 structures.

\section{Pathogenic APP-C99 mutations are more destabilizing in complex with $\gamma$-secretase}

As discussed above, all four methods indicate destabilization in APP-C99 and also consistently suggest more destabilization in the complex than in the free substrate, regardless of structural input curation. To test whether this finding is statistically significant, we compared the two cases specifically using a t-test with a zero hypothesis of identical average $\Delta \Delta \mathrm{G}_{\text {MUT. }}$. Most random mutations tend to destabilize, but in the relative comparison of the same protein (APPC99) in two different environments, the results are substantially more likely to be significant due to error cancellations of the methods.

Figures 3a-d shows the full distributions of $\Delta \Delta \mathrm{G}_{\mathrm{MUT}}$ values obtained for the model of apoAPP-C99 (2LLM) and when bound to $\gamma$-secretase (holo-APP-C99, 6IYC) for the four studied methods. Three of the methods indicate that the mutations are more destabilizing when in the context of the protein complex (except I-Mutant3.0). The t-test for same mean $\Delta \Delta \mathrm{G}_{\mathrm{MUT}}$ of the apo- and holo-structures showed significant results (95\% confidence level) for the holostructures using three of the methods (except I-Mutant3.0) (Supplementary Tables S3-S7). The mean $\Delta \Delta \mathrm{G}_{\mathrm{MUT}}$ values for the apo-structure were $0.35,0.47,0.46$ and $0.97 \mathrm{kcal} / \mathrm{mol}$ using FoldX, POPMUSIC, mCSM, and I-Mutant3.0, respectively. The corresponding mean values for the holo-structure were 1.21, 1.09, 0.89, and $0.80 \mathrm{kcal} / \mathrm{mol}$. Even though I-Mutant3.0 did not produce significantly larger destabilization in the holo-structure, it did produce substantial 
destabilization in both structures caused by mutation. For FoldX, a p-value (one-tailed) of 0.06 was obtained; when one major outlier was removed from the apo- and holo-dataset (Supplementary Table S4), the p-value was 0.02. The p-values for POPMUSIC, mCSM Stability and I-Mutant3.0 were $0.01,0.02$ and 0.14 . Accordingly, we conclude that there is consensus among the methods that fAD mutations tend to destabilize the complex more than the free separate proteins.

\section{APP-C99-mutations reduce the binding affinity to $\gamma$-secretase}

$\gamma$-secretase sequentially cuts APP-C99 to form A $\beta$ peptides of varying length[8-11]. The transmembrane segment of APP-C99 (C83 in 6IYC[34]) interacts closely with the catalytic PS1 subunit, and we thus expect that the mutations will affect the free and bound states differently. A larger destabilization in the complex could imply that the mutations reduce the substrate binding affinity, which would affect the retention time and thus extent of cleavage of APP-C99, which controls the $A \beta_{42} / A \beta_{42}$ ratio according to the FIST model[27,28]. This model is supported by the measurements of reduced enzyme-substrate interactions[62] and by MD simulations of wild-type $\gamma$-secretase in the membrane bound to full APP-C99[26], which binds more weakly than C83 used in the experimental structure[34]. We thus hypothesized that the fAD mutations may affect the Michaelis constant $K_{M}$ of the $\gamma$-secretase-APP complex since $\mathrm{K}_{\mathrm{M}}$ scales with the binding affinity of the substrate in the enzymatically active pose[26,63,64]. 

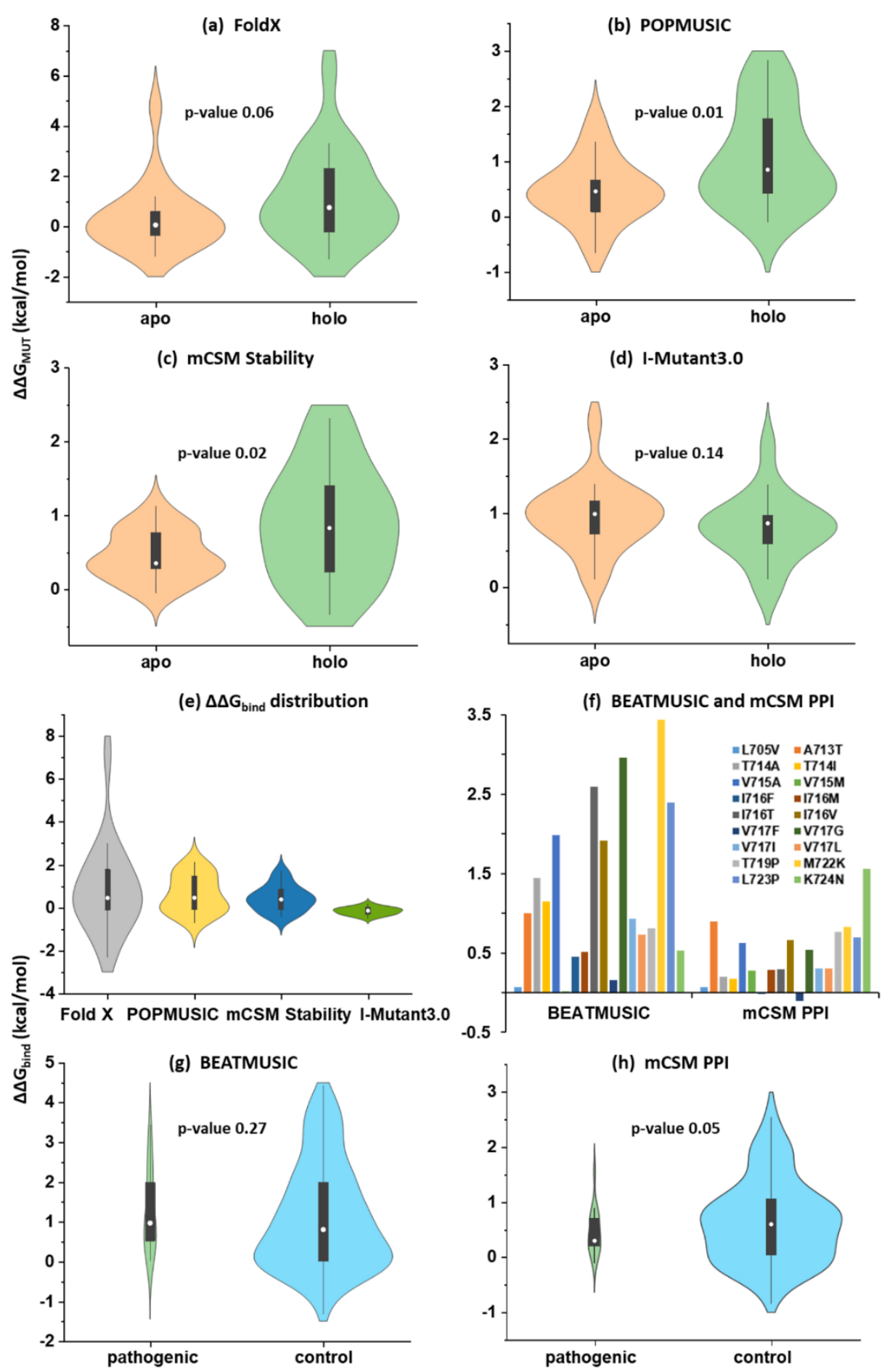

Figure 3. Distribution of stability effects ( $\Delta \Delta G_{M U T}$ ) of transmembrane APP-C99 mutations. Results are shown for C99 alone (apo-APP-C99, 2LLM) and in complex with $\gamma$ secretase (holo-APP-C99, 6IYC). The white dots represent the median (positive $\Delta \Delta \mathrm{G}_{\mathrm{MUT}}$ values are destabilizing). a) FoldX. b) POPMUSIC. c) mCSM Stability. d) I-Mutant3.0. e)

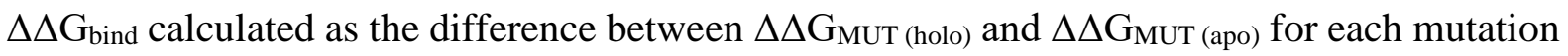
using FoldX, POPMUSIC, mCSM Stability and I-Mutant3.0. f) $\Delta \Delta \mathrm{G}_{\text {bind }}$ of mutation calculated using BEATMUSIC and mCSM PPI with structure 6IYC. g) Comparison of $\Delta \Delta \mathrm{G}_{\text {bind }}$ for APPC99 mutations vs. 202 control mutations using BEATMUSIC; h) Same as g) using mCSM PPI. 
To test this hypothesis, we calculated the distribution of the difference between the $\Delta \Delta \mathrm{G}_{\text {MUT }}$ energies of the holo (6IYC) and apo-APP-C99 (2LLM) structures $\left(\Delta \Delta \mathrm{G}_{\text {bind }}=\right.$ $\Delta \Delta \mathrm{G}_{\mathrm{MUT}(\mathrm{holo})}-\Delta \Delta \mathrm{G}_{\mathrm{MUT}(\mathrm{apo})}$; Figure 3e). These calculations consistently suggest a weakened enzyme-substrate interaction upon mutation for all the four methods. To test this finding further, we calculated the change in binding affinity due to pathogenic APP-C99 mutations in the holo-structure (6IYC) using two methods, BEATMUSIC and mCSM PPI, which were specifically developed for this purpose. Both these methods consistently showed a decrease in binding affinity/interaction for almost all pathogenic mutations (except I716F and V717F using mCSM PPI), Figure 3f. These results show that the pathogenic transmembrane APP-C99 mutations consistently tend to decrease the binding affinity and interaction of APP-C99 with $\gamma$-secretase, as also implied by the larger destabilization seen for the complex than the free state of APP-C99, in support of the FIST model[28]. Importantly, in addition to agreeing on this consensus despite major differences in the methods, the energy effects obtained by the stability predictors (Figure 2 and Figure 3a-3d) and those from the binding affinity methods (Figure 3f) are similar, typically 0-3 kcal/mol, although the BEATMUSIC estimates tend to be larger.

Since the 202 random control mutations also tend to decrease binding affinity, the loss of affinity was not significantly different for pathogenic and random mutations in the APP-C99 transmembrane domain (Figures 3g and $\mathbf{3 h}$ and Supplementary Tables S8 and S9). In fact, when using mCSM PPI, random mutations destabilized the complex significantly more than the pathogenic mutations. This is consistent with the fact that essentially all TM mutations in APP-C99 impair substrate binding due to their localization at the exact optimized substrate binding pocket[34]. This makes APP-C99 mutations distinct from PS1 mutations, which tend to delocalize over a wide area, and we conclude that any natural variation in this part of APPC99 will be likely to destabilize affinity towards the optimized binding site.

The $\mathrm{K}_{\mathrm{M}}$ of $\gamma$-secretase is unusually large $(0.2 \mathrm{mM})$ for a substrate the size of APP-C99 and the $\mathrm{k}_{\mathrm{cat}}$ has been reported to be very small $\left(0.0012 \mathrm{~s}^{-1}\right)$ [25]. Since substrate cleavage by $\gamma$ secretase is also sloppy, producing products of variable length, an impairment of the $\mathrm{K}_{\mathrm{M}}$ of pathogenic APP-C99 mutants, as found above, will probably reduce the trimming of APP-C99 and increase the $A \beta_{42} / A \beta_{40}$ ratio[65]. We note that mutations may also lower $K_{M}$ to produce overload of A $\beta$, as seen e.g. for the Swedish APP-double mutant, but it is a rare exception to the tendency of impaired substrate-enzyme interaction. Both mechanisms may relate to $\mathrm{AD}$, 
since the balance of $A \beta$ isoform may be more important[9,32] than simply overload as emphasized by the original amyloid cascade hypothesis[11].

\section{PS1 mutations strongly reduce protein stability but not APP-C99 affinity}

To analyze whether pathogenic PS1 mutations exhibit a similar effect on the binding affinity of APP-C99, we analyzed these mutations in the same way, in both the apo (5FN2) and holo (6IYC) states. Notably, we found similar destabilization in both states (Supplementary Figure S8), with no significant difference for the two structures (Supplementary Tables S10-S13). This implies that the PS1 mutations substantially destabilize the protein but do so to the same extent in both the apo- and holo-structures, assuming that the experimental structures are in the pathogenically relevant states. The conformational changes upon substrate binding are substantial in terms of geometry[34], but we show that the differences translate into small energy differences for the PS1 mutations, in stark contrast to the APP mutations, indicating that they may work by two different mechanisms.

According to our analysis, PS1 mutations do not cause fAD by reducing APP-C99 substrate affinity as seems the case for the APP mutations. However, PS1 mutations consistently reduce the stability of both apo- and holo-structures, and this loss of stability could be a direct, but distinct, cause of disease. To confirm that this destabilization is significant we used a t-test to compare the $\Delta \Delta \mathrm{G}_{\mathrm{MUT}}$ of pathogenic PS1-TM mutations against a control set of all possible mutations in all sites that are naturally variable as deduced from sequence alignment of PS1 to related proteins. As explained in Methods, we propose this control data set as the golden-standard for testing disease hypotheses when a critical amount of clinically confirmed non-pathogenic variants is not available, as is the case here.

We note that randomly introduced mutations in proteins tend to be destabilizing by perhaps $1 \mathrm{kcal} / \mathrm{mol}$ on average [66], reflecting the fact that protein stability is an evolutionarily optimized property that is impaired by random perturbation. It follows that most computational stability estimators will tend to produce more destabilization than stabilization, and this can make conclusions of destabilization by disease-causing mutations uninteresting if not compared against a control set.

Figure 4 shows the evaluation of the $\Delta \Delta \mathrm{G}_{\mathrm{MUT}}$ energies of the PS1 pathogenic dataset (147 mutations) against the control set (3048 mutations). The average destabilization due to pathogenic mutations is significantly higher than for the control mutations in both the apo- 
structure (5FN2) and holo-structure (6IYC) using all the four distinct methods (Supplementary Tables S14-S21). For the apo-structure, average $\Delta \Delta \mathrm{G}_{\text {MUT }}$ values of 2.26, $1.01,1.10$, and $1.15 \mathrm{kcal} / \mathrm{mol}$ are estimated for the pathogenic mutations, significantly higher than for control dataset with values of 1.65 (p-value 0.01), 0.88 (p-value 0.03), 0.98 (p-value 0.04), and $0.99 \mathrm{kcal} / \mathrm{mol}$ (p-value 0.0002) when calculated using FoldX, POPMUSIC, mCSM, and I-Mutant3.0, respectively. Similar results were obtained with the holo-structure with even more significant p-values of 0.0005, 0.0004, 0.01, and 0.00002 for FoldX, POPMUSIC, mCSM, and I-Mutant3.0, respectively. Thus, the pathogenic PS1 mutations significantly destabilize the enzyme-substrate complex but also destabilize PS1 when APP-C99 is not bound; in both cases, the results are highly significant and robust to both variations in method choice and structure input. Because of this general destabilization effect, we expect less activity of mutant PS1, as has indeed been observed in assays by Sun et al.[29]

When we compared the $\Delta \Delta \mathrm{G}_{\mathrm{MUT}}$ energies of the APP-C99 control dataset (202 mutations) with the pathogenic dataset (18 mutations), we did not find any significant differences (Supplementary Tables S22-S29 and Figure S9). Again, this probably reflects the fact that all TM mutations in APP tend to impair substrate binding to the evolutionarily optimized binding site, because of their location in the exact substrate binding site[34].

The membrane environment may affect protein destabilization upon mutation. Since the methods used do not consider the membrane environment, we divided the APP-C99 mutation positions into two groups, those likely to interact with the membrane and those likely to mainly interact with PS1. To do this, we built a homogenous POPC lipid bilayer on the 6IYC structure. The residues forming direct contacts within $4 \AA$ of C83 were analyzed. Out of the total ten positions, nine exclusively interact with PS1 and one position, L705, where only one mutation L705V occurs, interacts with both PS1 and POPC. Therefore, the APP-C99 mutations studied in this work are in contact with PS1 and not very affected by the presence of a membrane.

For PS1 (6IYC), we identified the mutation positions forming direct contact with the POPC bilayer. In total, the studied 147 mutations occur at 85 positions, out of which 25 positions directly interact with the POPC membrane (Supplementary Table S2). These 25 positions included 37 out of 147 mutations. This shows that most of the transmembrane pathogenic mutations (107 out of 147 mutations) occurred at positions that do not directly interact with the membrane, but that the overall membrane-protein system plays a larger role in understanding PS1 mutations than APP mutations, as also expected from previous studies $[67,68]$. Since the membrane coordinates are not generally present in the experimental 
cryo-EM structures, this is one of the major rationales for studying these proteins by molecular dynamics in the membrane, where the membrane creates a two-state situation in the holo-state which largely explains the sloppy APP-C99 cleavage.[26]
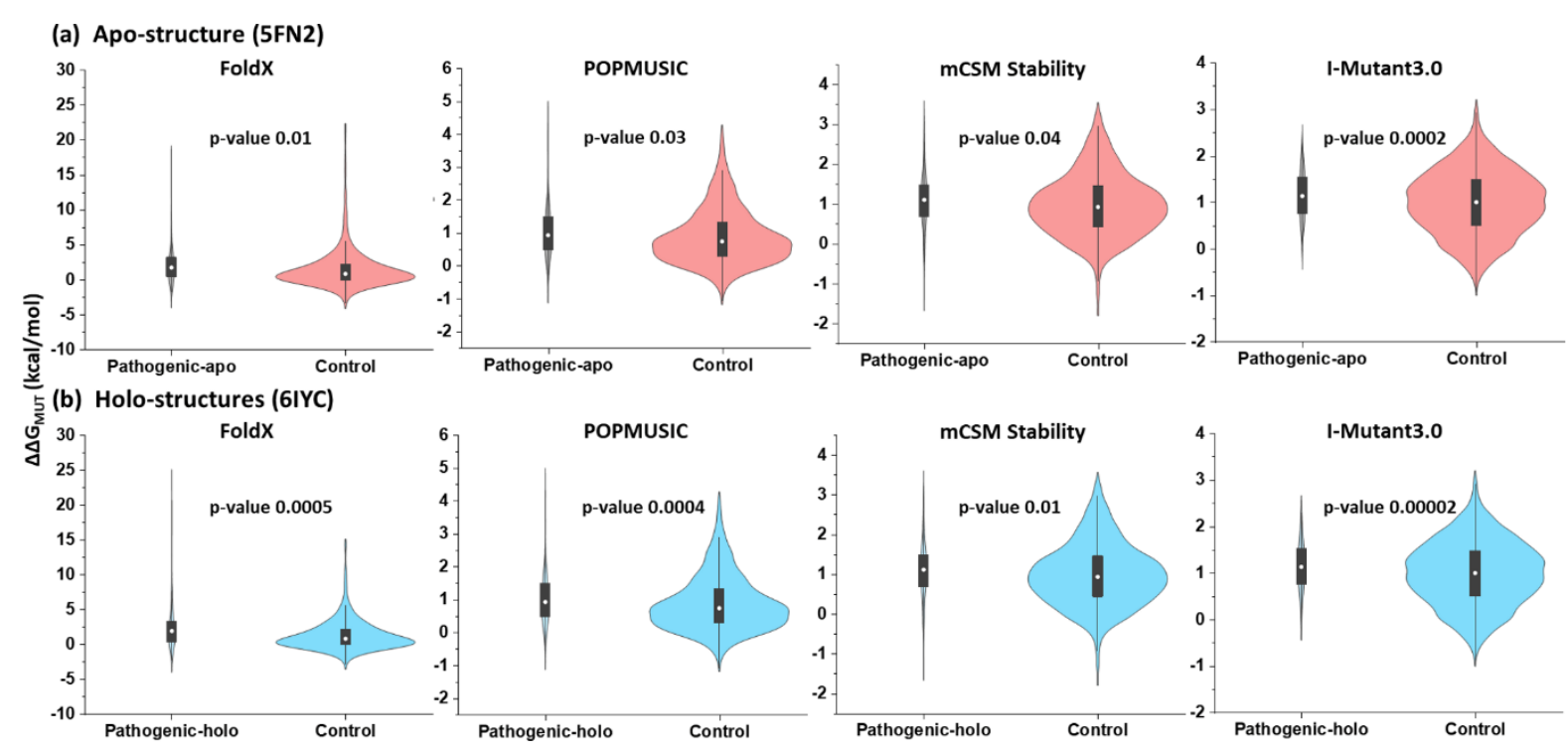

Figure 4. $\Delta \Delta G_{\text {MUT }}$ effects (kcal/mol) of 147 pathogenic transmembrane PS1 mutations and 3048 control mutations, calculated using four methods. The white dot inside the box and whisker plots shows the median. (a) Apo-structure (5FN2). (b) Substrate (C83)-bound holo-structure (6IYC).

\section{$A \beta_{42} / A \beta_{40}$ ratio correlates with $\Delta \Delta G_{M U T}$ of pathogenic PS1 TM mutations in the holo state}

To understand whether the much explored $A \beta_{42} / A \beta_{40}$ ratios of PS1 mutations correlate with mutant stability, we compared available $A \beta_{42} / A \beta_{40}$ ratios[29] for the studied mutations (Supplementary Table S2) with the $\Delta \Delta \mathrm{G}_{\mathrm{MUT}}$ energies of both the PS1 apo-structure (5FN2) and holo-structure (6IYC) (Supplementary Figures S10-S13). 56 A $\beta_{42} / A \beta_{40}$ ratios were available for mutations in sites covered by the structures. The $A \beta_{42} / A \beta_{40}$ ratios resemble equilibrium constants and we thus converted them to the $\log _{10}$ scale in order to compare to the free energies. For the PS1 apo-structure, the correlation was not significant for any of the methods (Figure S10), although mCSM was significant after removing one outlier (Figure S11). This suggests that the stability effect on the apo state of PS1 is not the cause of the increased $A \beta_{42} / A \beta_{40}$ ratio. 


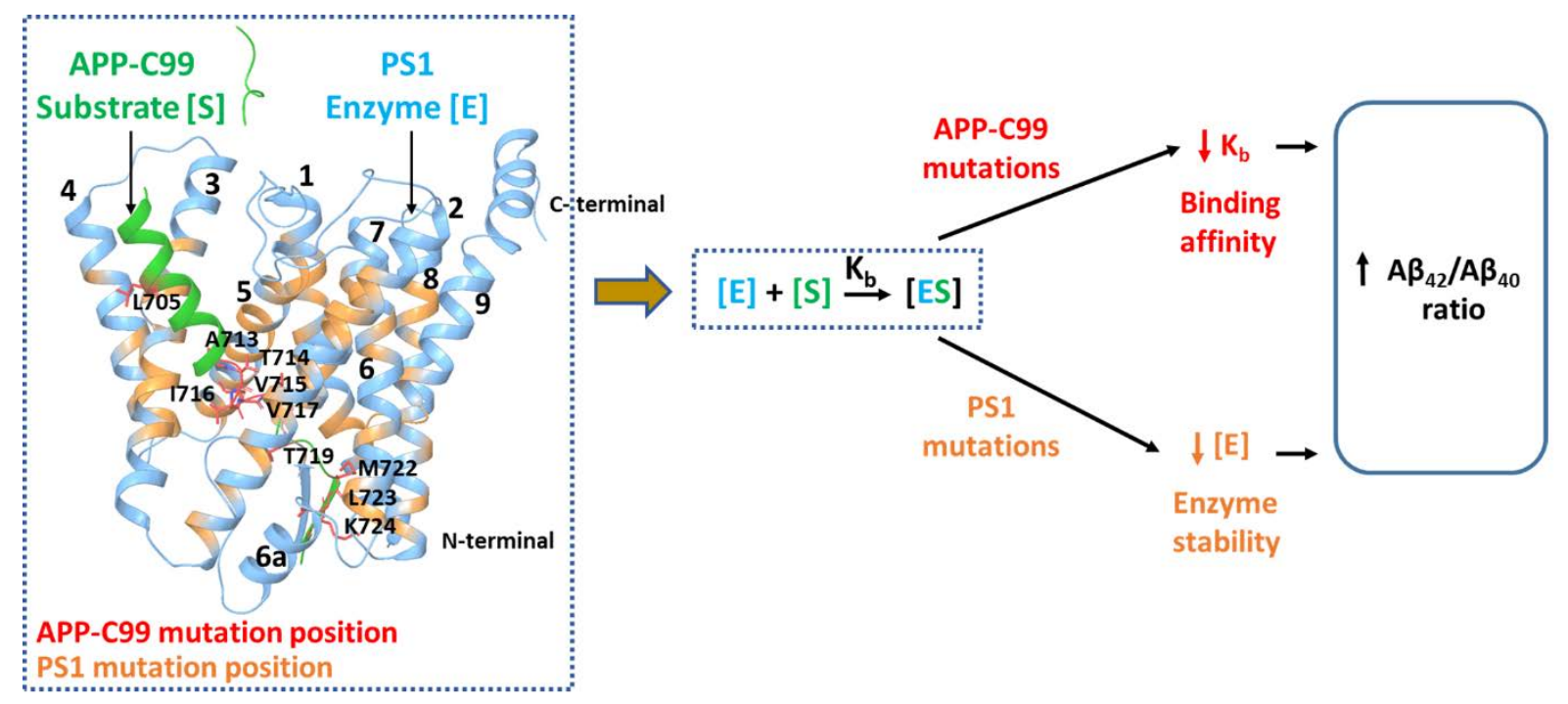

Figure 5. The proposed two different mechanisms of action of AD-associated pathogenic APP-C99 and PS1 transmembrane mutations. APP-C99 (C83) is shown in green ribbons with mutation positions represented in red colored stick model and labelled with residue information. The PS1 transmembrane segments are shown in cyan ribbons and numbered from 1-9, with mutation positions colored as orange. The substrate APP-C99 (S) shows a decrease in binding affinity $\left(\mathrm{K}_{\mathrm{b}}\right)$ to PS1 (E) upon mutations. Mutations in PS1 decreases the protein/enzyme (E) folding stability. Both these mechanisms may probably cause an early release of the substrate/product thereby increasing the $A \beta_{42} / A \beta_{40}$ ratios.

For the PS1 holo-structure, the correlation was significant (95\% confidence) after removal of one outlier for all methods except POPMUSIC (Figures S12 and S13). FoldX, mCSM Stability, and I-Mutant produced $\mathrm{R}^{2}$-values of 0.14 (p-value 0.004 ), 0.12 (p-value 0.01), and 0.08 (p-value 0.03). POPMUSIC instead gave $\mathrm{R}^{2}=0.06$, with $\mathrm{p}$-value 0.07 . Considering the noise in the experimental assayed data[29], the structural resolution and associated noise[34], and the $1 \mathrm{kcal} / \mathrm{mol}$ random errors in the computational methods[56,58,61], the small $\mathrm{R}$ values are expected, yet the p-values are significant and the direction of correlation is meaningful and largely independent of method. These results indicate that when using the new holo-structure of $\gamma$-secretase as input[34], $A \beta_{42} / A \beta_{40}$ ratios relate to the stability effects caused by mutations as hypothesized previously[28], and in general, $A \beta_{42} / A \beta_{40}$ increases with decreased protein stability. The better correlation for the holo structure probably reflects that this is the state that is actually responsible for producing $A \beta$ and thus probably quantifies a real chemical impact of mutation on the different conformations of PS1 in holo (6IYC) vs. apo (5FN2) induced by the substrate binding. For the studied APP-C99 mutations, the number of experimental 
$\mathrm{A} \beta_{42} / \mathrm{A} \beta_{40}$ ratios are too few (Supplementary Table S1) to make a meaningful test, and these correlations were accordingly not significant (Supplementary Figure S14).

Based on the structure-based calculations performed using six methods in this work, the probable mechanisms of action of the APP-C99 and PS1 transmembrane pathogenic mutations in relation to $\gamma$-secretase are summarized in Figure 5. Both types of mutations destabilize the protein. However, most APP-C99 mutations destabilize the complex more, due to their location in the substrate binding pocket, and thus reduce substrate affinity $\left(\mathrm{K}_{\mathrm{b}}\right)$, which leads to earlier release of the substrate or its intermediate products, imprecise cleavage, reduced trimming, less total $A \beta$, and relatively more of the long $A \beta$ peptides caused by less average trimming of each APP-C99 substrate. An exception to this tendency is the Swedish mutation, which increases total A $\beta$ by affecting $\beta$-secretase rather than $\gamma$-secretase. Some mutations in PS1 do the same as seen from the data. They generally destabilize the protein to produce a less stable and active enzyme, which, as shown by Sun et al. also correlates with increased $A \beta_{42} / A \beta_{40}$ ratios[29]. The destabilization of PS1 mutations was previously suggested to correlate with $A \beta_{42} / A \beta_{40}$ ratio by computation[28], and later confirmed experimentally for five mutants[62]. Based on the strong consensus of the diverse methods and trusting the experimental structures as input, we conclude that this mechanism is not isolated to a few mutants but is almost universal.

To obtain an overview of the qualitative effects of the fAD mutations, the pathogenic TM mutations in APP-C99 and PS1 were categorized into four groups based on $\Delta \Delta \mathrm{G}_{\mathrm{MUT}}$ as (i) destabilizing both the apo- and holo- $\gamma$-secretase, (ii) stabilizing both apo- and holo- $\gamma$-secretase, (iii) destabilizing apo- and stabilizing holo- $\gamma$-secretase, and (iv) stabilizing apo- and destabilizing holo- $\gamma$-secretase (Supplementary Figure S15 and Tables S30 and S31). By far the majority of mutations are predicted to be destabilizing in both the apo and holo structures (category i), with only a few mutations belonging to the other three categories (ii, iii and iv). We expect false positives considering the uncertainties in the theoretical methods and cryo-EM structures, but the tendency is very strong regardless. For PS1, 110-144 of the 147 pathogenic TM mutations belong to category i, depending on method used, and 95 of these mutations, a clear majority, were common to the methods despite their diversity (Table S31).

\section{Conclusions}

In this work, we studied the impact of fAD-causing mutations in the transmembrane regions of PS1 and APP-C99 by four very different computational protein-stability methods and two 
substrate affinity methods. We used the available structures of a fragment of APP-C99 alone, of $\gamma$-secretase without substrate bound (apo-state), and $\gamma$-secretase bound to APP-C83 (holostate), as recently published[34]. We considered all mutations that occurred in sites common to all available experimental structures, which provide a non-arbitrary test of the impact of mutations on the different structural states. The main conclusions of the study are:

(1) Pathogenic transmembrane mutations in APP-C99 destabilize the protein both in the free form and in complex with $\gamma$-secretase (holo-state), but significantly more in the latter. Thereby, APP-C99 mutations consistently decrease the affinity of the substrate for $\gamma$-secretase as confirmed by two independent methods developed specifically for this purpose.

(2) Pathogenic transmembrane mutations in PS1 destabilize both the apo- and holostructures to a similar extent.

(3) The PS1 pathogenic transmembrane mutations consistently destabilize PS1 more than other possible mutations in variable sites of the protein, with very significant p-values when using both the apo- and holo-structures.

Our findings suggest that the PS1 mutations are too scattered even in the TM part of PS1 to consistently affect substrate affinity, whereas they consistently reduce protein stability of both apo- and holo states. In contrast, the APP mutations are located very much in the site of interaction with PS1 and thus exert a direct effect on substrate binding affinity. This difference in localization can explain our findings that the two types of fAD mutations work in distinct ways, and may also potentially explain why the clinical and biochemical manifestations of the two types of fAD mutations differ to some extent.

\section{Acknowledgements}

The Danish Council for Independent Research | Natural Sciences (DFF), grant case 701600079B, is acknowledged for supporting this work.

\section{Supporting Information}

The supporting information file contains the dataset of APP-C99 and PS1 transmembrane mutations, the associated data for $A \beta_{42} / A \beta_{40}$ ratios, the statistical t-test data of the analysis, sorting of mutations (qualitative analysis), the data of variable mutation sites (control datasets), 
the data of structural sensitivity tests, PS1 apo versus holo analysis, APP-C99 pathogenic versus control analysis, and the relation between $A \beta_{42} / A \beta_{40}$ ratios and $\Delta \Delta G_{\text {MUT }}$ values.

\section{ORCID}

$\mathrm{RM}: \underline{0000-0001-6010-1514}, \mathrm{KPK}: \underline{0000-0002-6754-7348}$

\section{References}

[1] J.F. Dartigues, Alzheimer's disease: a global challenge for the 21st century, Lancet Neurol. 8 (2009) 1082-1083. doi:10.1016/s1474-4422(09)70298-4.

[2] M. Prince, R. Bryce, E. Albanese, A. Wimo, W. Ribeiro, C.P. Ferri, The global prevalence of dementia: a systematic review and metaanalysis., Alzheimers. Dement. 9 (2013) 63-75.e2. doi:10.1016/j.jalz.2012.11.007.

[3] D.M. Holtzman, J.C. Morris, A.M. Goate, Alzheimer's disease: The challenge of the second century, Sci. Transl. Med. 3 (2011) 77sr1. doi:10.1126/scitranslmed.3002369.

[4] E. Nichols, et al. Global, regional, and national burden of Alzheimer's disease and other dementias, 1990-2016: a systematic analysis for the Global Burden of Disease Study 2016, Lancet Neurol. 18 (2019) 88-106. doi:10.1016/S1474-4422(18)30403-4.

[5] K. Blennow, M.J. de Leon, H. Zetterberg, Alzheimer’s disease, Lancet. 368 (2015) 387-403. doi:10.1016/S0140-6736(06)69113-7.

[6] M. Prince, A. Comas-Herrera, M. Knapp, M. Guerchet, M. Karagiannidou, World Alzheimer Report 2016 Improving healthcare for people living with dementia. Coverage, Quality and costs now and in the future, Alzheimer’s Dis. Int. (2016) 1-140.

[7] G. He, W. Luo, P. Li, C. Remmers, W.J. Netzer, J. Hendrick, K. Bettayeb, M. Flajolet, F. Gorelick, L.P. Wennogle, P. Greengard, Gamma-secretase activating protein is a therapeutic target for Alzheimer’s disease, Nature. 467(7311) (2010) 95. doi:10.1038/nature09325.

[8] M.S. Wolfe, $\gamma$-Secretase as a Target for Alzheimer's Disease, Curr. Top. Med. Chem. 2 (2002) 371-383. 
[9] K.P. Kepp, Ten Challenges of the Amyloid Hypothesis of Alzheimer's Disease, J. Alzheimer’s Dis. 55 (2017) 447-457. doi:10.3233/JAD-160550.

[10] J.A. Hardy, G.A. Higgins, Alzheimer's disease: the amyloid cascade hypothesis., Science (80-. ). 256 (1992) 184-185.

[11] J. Hardy, Alzheimer's disease: The amyloid cascade hypothesis - An update and reappraisal, J. Alzheimer’s Dis. 9 (2006) 151-153.

[12] W.T. Kimberly, M.J. LaVoie, B.L. Ostaszewski, W. Ye, M.S. Wolfe, D.J. Selkoe, Gamma-secretase is a membrane protein complex comprised of presenilin, nicastrin, Aph-1, and Pen-2., Proc. Natl. Acad. Sci. U. S. A. 100 (2003) 6382-6387. doi:10.1073/pnas.1037392100.

[13] X. Bai, C. Yan, G. Yang, P. Lu, L. Sun, R. Zhou, S.H.W. Scheres, Y. Shi, An atomic structure of human $\gamma$-secretase, Nature. 525 (2015) 212-218. doi:10.1038/nature14892.

[14] B. De Strooper, T. Iwatsubo, M.S. Wolfe, Presenilins and $\gamma$-secretase: structure, function, and role in Alzheimer Disease., Cold Spring Harb. Perspect. Med. 2 (2012) a006304. doi:10.1101/cshperspect.a006304.

[15] A.Y. Kornilova, F. Bihel, C. Das, M.S. Wolfe, The initial substrate-binding site of $\gamma$ secretase is located on presenilin near the active site, Proc. Natl. Acad. Sci. 102 (2005) 3230-3235. doi:10.1073/pnas.0407640102.

[16] D.J. Selkoe, Presenilin, Notch, and the genesis and treatment of Alzheimer's disease, Proc. Natl. Acad. Sci. 98 (2002) 11039-11041. doi:10.1073/pnas.211352598.

[17] J. Kinoshita, T. Clark, Alzforum, in: Neuroinformatics. Humana Press, 2008: pp. 365381. doi:10.1007/978-1-59745-520-6_19.

[18] M. Cruts, J. Theuns, C. Van Broeckhoven, Locus-specific mutation databases for neurodegenerative brain diseases, Hum. Mutat. 33 (2012) 1340-1344. doi:10.1002/humu.22117.

[19] M. Bentahir, O. Nyabi, J. Verhamme, A. Tolia, K. Horre, J. Wiltfang, H. Esselmann, B. De Strooper, Presenilin clinical mutations can affect gamma-secretase activity by different mechanisms., J. Neurochem. 96 (2006) 732-742. doi:10.1111/j.14714159.2005.03578.x.

[20] D.R. Borchelt, G. Thinakaran, C.B. Eckman, M.K. Lee, F. Davenport, T. Ratovitsky, 
C.M. Prada, G. Kim, S. Seekins, D. Yager, H.H. Slunt, R. Wang, M. Seeger, A.I. Levey, S.E. Gandy, N.G. Copeland, N.A. Jenkins, D.L. Price, S.G. Younkin, S.S. Sisodia, Familial Alzheimer's disease-linked presenilin 1 variants elevate Abeta142/1-40 ratio in vitro and in vivo., Neuron. 17 (1996) 1005-1013. doi:10.1016/S08966273(00)80230-5.

[21] O. Murayama, T. Tomita, N. Nihonmatsu, M. Murayama, X. Sun, T. Honda, T. Iwatsubo, A. Takashima, Enhancement of amyloid $\beta 42$ secretion by 28 different presenilin 1 mutations of familial Alzheimer’s disease, Neurosci. Lett. 265 (1999) 6163. doi:10.1016/S0304-3940(99)00187-1.

[22] C. De Jonghe, C. Esselens, S. Kumar-Singh, K. Craessaerts, S. Serneels, F. Checler, W. Annaert, C. Van Broeckhoven, B. De Strooper, Pathogenic APP mutations near the gamma-secretase cleavage site differentially affect Abeta secretion and APP Cterminal fragment stability., Hum. Mol. Genet. 10 (2001) 1665-1671. doi:10.1093/hmg/10.16.1665.

[23] Y. Yan, C. Wang, $A \beta 42$ is More Rigid than $A \beta 40$ at the $C$ Terminus: Implications for A $\beta$ Aggregation and Toxicity, J. Mol. Biol. 364 (2006) 853-862.

doi:10.1016/j.jmb.2006.09.046.

[24] N. Tang, K.P. Kepp, A $\beta 42 / A \beta 40$ ratios of presenilin 1 mutations correlate with clinical onset of Alzheimer’s disease, J. Alzheimer’s Dis. 66 (2018) 939-945.

[25] F. Kamp, E. Winkler, J. Trambauer, A. Ebke, R. Fluhrer, H. Steiner, Intramembrane proteolysis of $\beta$-amyloid precursor protein by $\gamma$-secretase is an unusually slow process, Biophys. J. 108 (2015) 1229-1237. doi:10.1016/j.bpj.2014.12.045.

[26] B. Dehury, N. Tang, K.P. Kepp, Molecular Dynamics of C99-Bound $\gamma$-Secretase Reveal Two Binding Modes with Distinct Compactness, Stability, and Active-Site Retention: Implications for A $\beta$ Production., Biochem. J. (2019) BCJ20190023. doi:10.1042/BCJ20190023.

[27] A.K. Somavarapu, K.P. Kepp, Membrane Dynamics of $\gamma$-Secretase Provides a Molecular Basis for $\beta$-Amyloid Binding and Processing, ACS Chem. Neurosci. 8 (2017) 2424-2436. doi:10.1021/acschemneuro.7b00208.

[28] A.K. Somavarapu, K.P. Kepp, Loss of stability and hydrophobicity of presenilin 1 mutations causing Alzheimer’s Disease., J. Neurochem. 137 (2016) 101-111. 
doi:10.1111/jnc.13535.

[29] L. Sun, R. Zhou, G. Yang, Y. Shi, Analysis of 138 pathogenic mutations in presenilin1 on the in vitro production of $A \beta 42$ and $A \beta 40$ peptides by $\gamma$-secretase, Proc. Natl. Acad. Sci. 114 (2016) E476-E485. doi:10.1073/pnas.1618657114.

[30] N. Tang, A.K. Somavarapu, K.P. Kepp, Molecular Recipe for $\gamma$-Secretase Modulation from Computational Analysis of 60 Active Compounds, ACS Omega. 3 (2018) 18078-18088. doi:10.1021/acsomega.8b02196.

[31] J. Shen, R.J. Kelleher, The presenilin hypothesis of Alzheimer's disease: evidence for a loss-of-function pathogenic mechanism., Proc. Natl. Acad. Sci. U. S. A. 104 (2007) 403-409. doi:10.1073/pnas.0608332104.

[32] K.P. Kepp, Alzheimer's disease due to loss of function: A new synthesis of the available data, Prog. Neurobiol. 143 (2016) 36-60.

doi:10.1016/j.pneurobio.2016.06.004.

[33] K.P. Kepp, A quantitative model of human neurodegenerative diseases involving protein aggregation, Neurobiol. Aging. 80 (2019) 46-55. doi:https://doi.org/10.1016/j.neurobiolaging.2019.04.001.

[34] R. Zhou, G. Yang, X. Guo, Q. Zhou, J. Lei, Y. Shi, Recognition of the amyloid precursor protein by human $\gamma$-secretase, Science (80-. ). 363 (2019) eaaw0930. doi:10.1126/science.aaw0930.

[35] G. Yang, R. Zhou, Q. Zhou, X. Guo, C. Yan, M. Ke, J. Lei, Y. Shi, Structural basis of Notch recognition by human $\gamma$-secretase, Nature. 565 (2019) 192-197. doi:10.1038/s41586-018-0813-8.

[36] R. Guerois, J.E. Nielsen, L. Serrano, Predicting Changes in the Stability of Proteins and Protein Complexes: A Study of More Than 1000 Mutations, J. Mol. Biol. 320 (2002) 369-387. doi:10.1016/S0022-2836(02)00442-4.

[37] J. Schymkowitz, J. Borg, F. Stricher, R. Nys, F. Rousseau, L. Serrano, The FoldX web server: an online force field, Nucleic Acids Res. 33 (2005) W382-W388. doi:10.1093/nar/gki387.

[38] D. Gilis, M. Rooman, PoPMuSiC, an algorithm for predicting protein mutant stability changes: application to prion proteins., Protein Eng. 13 (2000) 849-856. 
http://www.ncbi.nlm.nih.gov/pubmed/11239084.

[39] Y. Dehouck, J.M. Kwasigroch, D. Gilis, M. Rooman, PoPMuSiC 2.1: a web server for the estimation of protein stability changes upon mutation and sequence optimality., BMC Bioinformatics. 12 (2011) 151. doi:10.1186/1471-2105-12-151.

[40] D.E. Pires, D.B. Ascher, T.L. Blundell, mCSM: predicting the effects of mutations in proteins using graph-based signatures, Bioinformatics. 30 (2013) 335-342. doi:10.1093/bioinformatics/btt691.

[41] E. Capriotti, P. Fariselli, R. Casadio, I-Mutant2.0: predicting stability changes upon mutation from the protein sequence or structure, Nucleic Acids Res. 33 (2005) W306W310.

[42] K.D. Nadezhdin, O. V Bocharova, E. V Bocharov, A. Arseniev, Structural and dynamic study of the transmembrane domain of the amyloid precursor protein., Acta Naturae. 3 (2011) 8.

[43] X. Bai, E. Rajendra, G. Yang, Y. Shi, S.H. Scheres, Sampling the conformational space of the catalytic subunit of human $\gamma$-secretase, Elife. 4 (2015) 551-560. doi:10.7554/eLife.11182.

[44] Schrödinger Release 2018-2: Protein Preparation Wizard; Epik, Impact; Schrödinger, LLC, New York, NY, 2018, (n.d.).

[45] M.A. Lomize, I.D. Pogozheva, H. Joo, H.I. Mosberg, A.L. Lomize, OPM database and PPM web server: Resources for positioning of proteins in membranes, Nucleic Acids Res. 40 (2012) D370-D376. doi:10.1093/nar/gkr703.

[46] S. Jo, T. Kim, V.G. Iyer, W. Im, CHARMM-GUI: A web-based graphical user interface for CHARMM, J. Comput. Chem. 29 (2008) 1859-1865. doi:10.1002/jcc.20945.

[47] D.R. Thal, W.S.T. Griffin, R.A.I. de Vos, E. Ghebremedhin, Cerebral amyloid angiopathy and its relationship to Alzheimer's disease, Acta Neuropathol. 115 (2008) 599-609. doi:10.1007/s00401-008-0366-2.

[48] W.D. Brenowitz, P.T. Nelson, L.M. Besser, K.B. Heller, W.A. Kukull, Cerebral amyloid angiopathy and its co-occurrence with Alzheimer's disease and other cerebrovascular neuropathologic changes, Neurobiol. Aging. 36 (2015) 2702-2708. 
doi:10.1016/j.neurobiolaging.2015.06.028.

[49] N. Tang, B. Dehury, K.P. Kepp, Computing the Pathogenicity of Alzheimer's Disease Presenilin 1 Mutations, J. Chem. Inf. Model. 59 (2019) 858-870. doi:10.1021/acs.jcim.8b00896.

[50] K.P. Kepp, Genotype-Property Patient-Phenotype Relations Suggest that Proteome Exhaustion Can Cause Amyotrophic Lateral Sclerosis, PLoS One. 10 (2015) e0118649. doi:10.1371/journal.pone.0118649.

[51] J. Thusberg, A. Olatubosun, M. Vihinen, Performance of mutation pathogenicity prediction methods on missense variants, Hum Mutat. 32 (2011). doi:10.1002/humu.21445.

[52] G.C.P. Schaafsma, M. Vihinen, Large differences in proportions of harmful and benign amino acid substitutions between proteins and diseases, Hum. Mutat. 38 (2017) 839-848.

[53] A. Marchler-Bauer, S.H. Bryant, CD-Search: Protein domain annotations on the fly, Nucleic Acids Res. 32 (2004) W327-331. doi:10.1093/nar/gkh454.

[54] E. Capriotti, R. Calabrese, R. Casadio, Predicting the insurgence of human genetic diseases associated to single point protein mutations with support vector machines and evolutionary information, Bioinformatics. 22 (2006) 2729-2734. doi:10.1093/bioinformatics/btl423.

[55] Y. Dehouck, J.M. Kwasigroch, M. Rooman, D. Gilis, BeAtMuSiC: Prediction of changes in protein-protein binding affinity on mutations., Nucleic Acids Res. 41 (2013) W333-W339. doi:10.1093/nar/gkt450.

[56] S. Khan, M. Vihinen, Performance of protein stability predictors., Hum. Mutat. 31 (2010) 675-684. doi:10.1002/humu.21242.

[57] J. Holm, P. Dasmeh, K.P. Kepp, Tracking evolution of myoglobin stability in cetaceans using experimentally calibrated computational methods that account for generic protein relaxation, Biochim. Biophys. Acta - Proteins Proteomics. 1864 (2016) 825-834. doi:http://dx.doi.org/10.1016/j.bbapap.2016.04.004.

[58] K.P. Kepp, Computing stability effects of mutations in human superoxide dismutase 1., J. Phys. Chem. B. 118 (2014) 1799-1812. doi:10.1021/jp4119138. 
[59] N.J. Christensen, K.P. Kepp, Accurate stabilities of laccase mutants predicted with a modified FoldX protocol, J. Chem. Inf. Model. 52 (2012) 3028-3042. doi:10.1021/ci300398z.

[60] V. Potapov, M. Cohen, G. Schreiber, Assessing computational methods for predicting protein stability upon mutation: good on average but not in the details., Protein Eng. Des. Sel. 22 (2009) 553-560. doi:10.1093/protein/gzp030.

[61] K.P. Kepp, Towards a "Golden Standard” for computing globin stability: Stability and structure sensitivity of myoglobin mutants, Biochim. Biophys. Acta - Proteins Proteomics. 1854 (2015) 1239-1248. doi:10.1016/j.bbapap.2015.06.002.

[62] M. Szaruga, B. Munteanu, S. Lismont, S. Veugelen, K. Horré, M. Mercken, T.C. Saido, N.S. Ryan, T. De Vos, S.N. Savvides, R. Gallardo, J. Schymkowitz, F. Rousseau, N.C. Fox, C. Hopf, B. De Strooper, Alzheimer's-Causing Mutations Shift A $\beta$ Length by Destabilizing $\gamma$-Secretase-A $\beta n$ Interactions, Cell. 170 (2017) 443-456.

[63] R. Mehra, A.S. Meyer, K.P. Kepp, Molecular dynamics derived life times of active substrate binding poses explain KM of laccase mutants, RSC Adv. 8 (2018) 3691536926. doi:10.1039/c8ra07138a.

[64] R. Mehra, J. Muschiol, A.S. Meyer, K.P. Kepp, A structural-chemical explanation of fungal laccase activity, Sci. Rep. 8 (2018) 17285.

[65] M.K. Tiwari, K.P. Kepp, $\beta$-Amyloid pathogenesis: Chemical properties versus cellular levels, Alzheimer’s Dement. 12 (2016) 184-194. doi:10.1016/j.jalz.2015.06.1895.

[66] N. Tokuriki, F. Stricher, J. Schymkowitz, L. Serrano, D.S. Tawfik, The stability effects of protein mutations appear to be universally distributed., J. Mol. Biol. 369 (2007) 1318-1332. doi:10.1016/j.jmb.2007.03.069.

[67] L. Dominguez, L. Foster, J.E. Straub, D. Thirumalai, Impact of membrane lipid composition on the structure and stability of the transmembrane domain of amyloid precursor protein, Proc. Natl. Acad. Sci. 113 (2016) E5281-E5287. doi:10.1073/pnas.1606482113.

[68] E. Winkler, F. Kamp, J. Scheuring, A. Ebke, A. Fukumori, H. Steiner, Generation of Alzheimer disease-associated amyloid $\beta 42 / 43$ peptide by $\gamma$-secretase can be inhibited directly by modulation of membrane thickness., J. Biol. Chem. 287 (2012) 21326-34. doi:10.1074/jbc.M112.356659. 